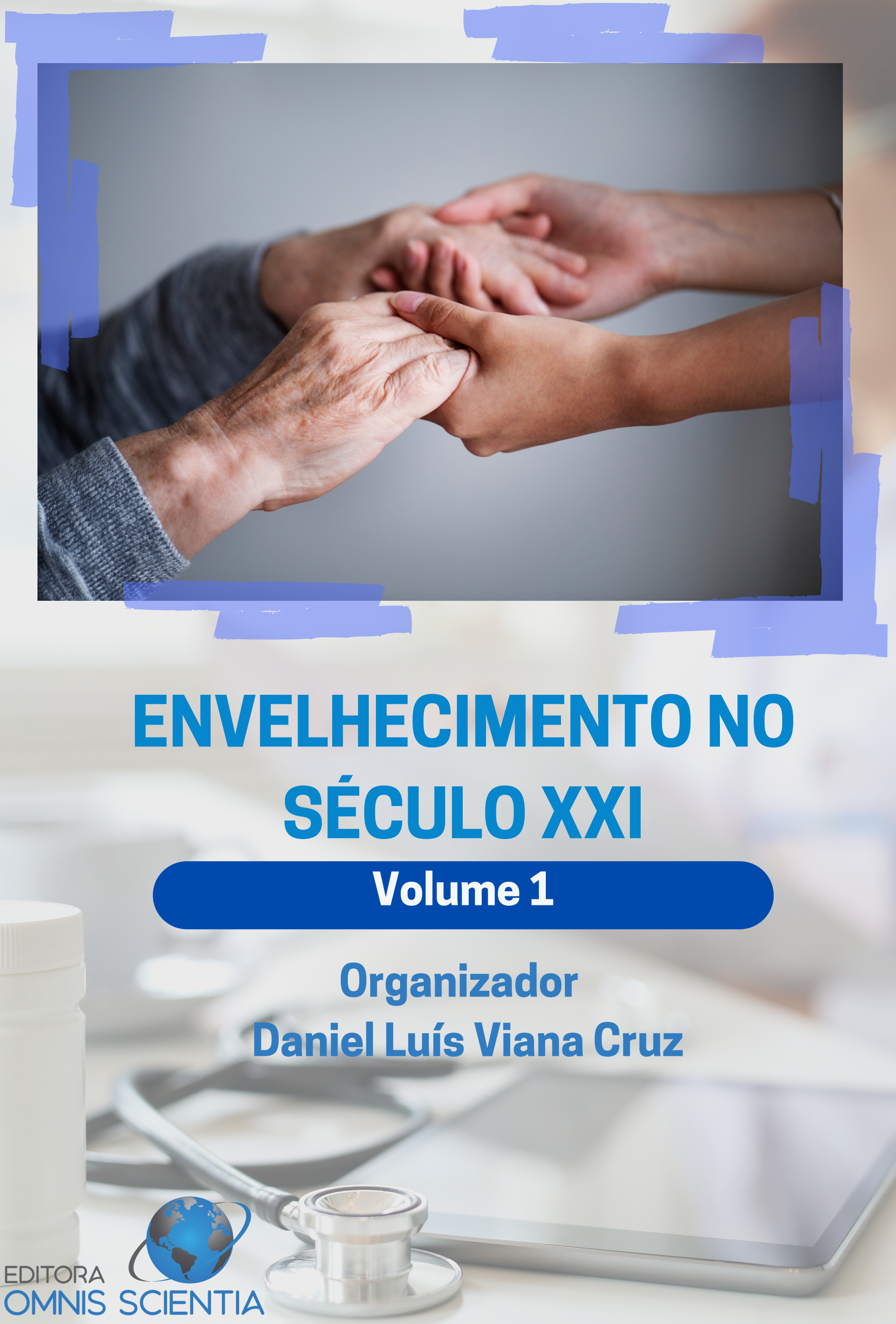




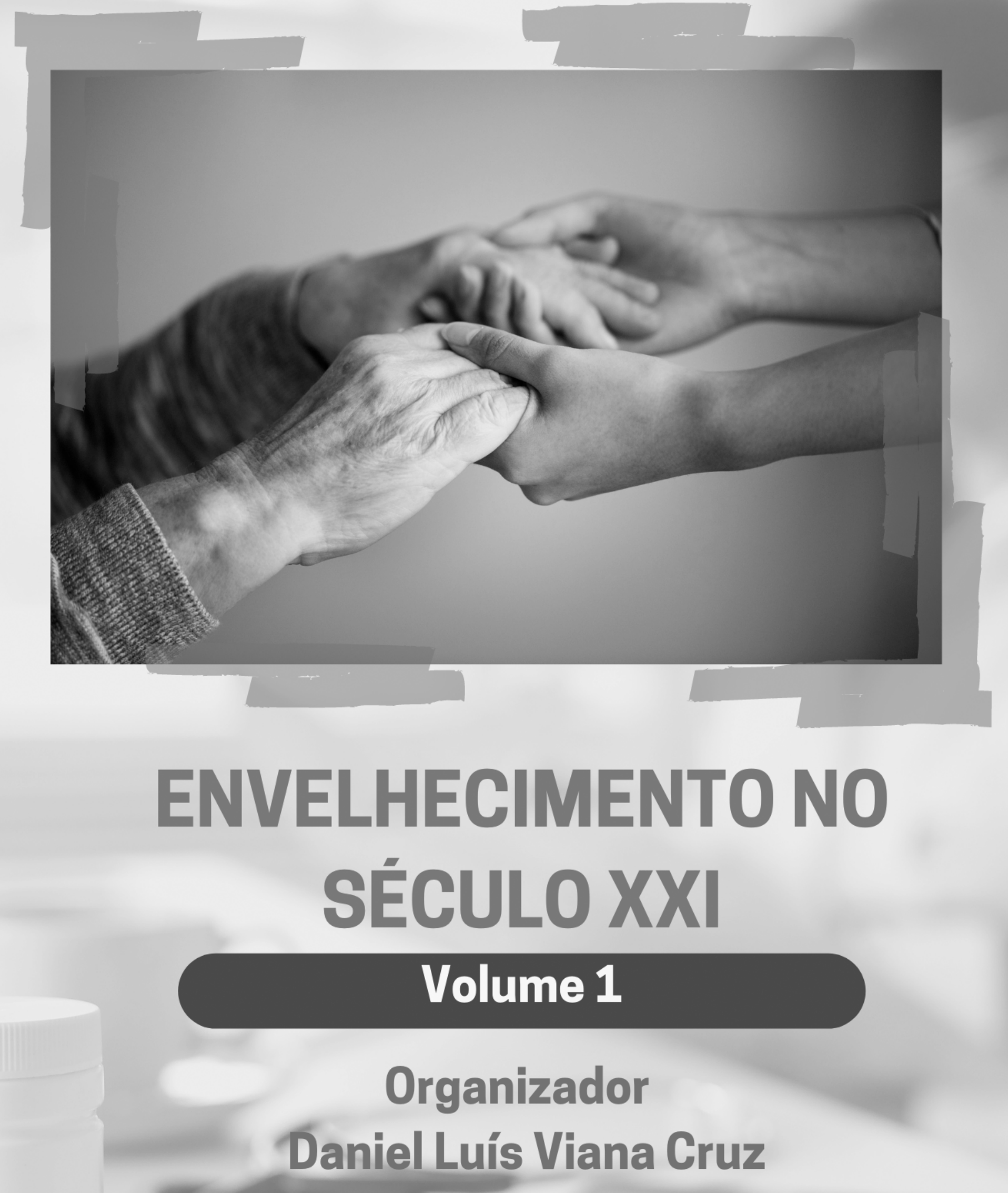

EDITORA

OMNIS SCIENTIA 
Editora Omnis Scientia

ENVELHECIMENTO NO SÉCULO XXI

Volume 1

$1^{a}$ Edição

TRIUNFO - PE 


\section{Editor-Chefe}

Me. Daniel Luís Viana Cruz

Organizador (a)

Me. Daniel Luís Viana Cruz

\section{Conselho Editorial}

Dra. Pauliana Valéria Machado Galvão

Dr. Wendel José Teles Pontes

Dr. Walter Santos Evangelista Júnior

Dr. Cássio Brancaleone

Dr. Plínio Pereira Gomes Júnior

Editores de Área - Ciências da Saúde

Dra. Camyla Rocha de Carvalho Guedine

Dr. Leandro dos Santos

Dr. Hugo Barbosa do Nascimento

Dra. Pauliana Valéria Machado Galvão

\section{Assistentes Editoriais}

Thialla Larangeira Amorim

Andrea Telino Gomes

\section{Imagem de Capa}

Freepik

Edição de Arte

Leandro José Dionísio

\section{Revisão}

Os autores

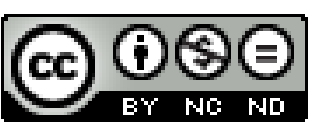

Este trabalho está licenciado com uma Licença Creative Commons - AtribuiçãoNãoComercial-SemDerivações 4.0 Internacional.

O conteúdo abordado nos artigos, seus dados em sua forma, correção e confiabilidade são de responsabilidade exclusiva dos autores. 


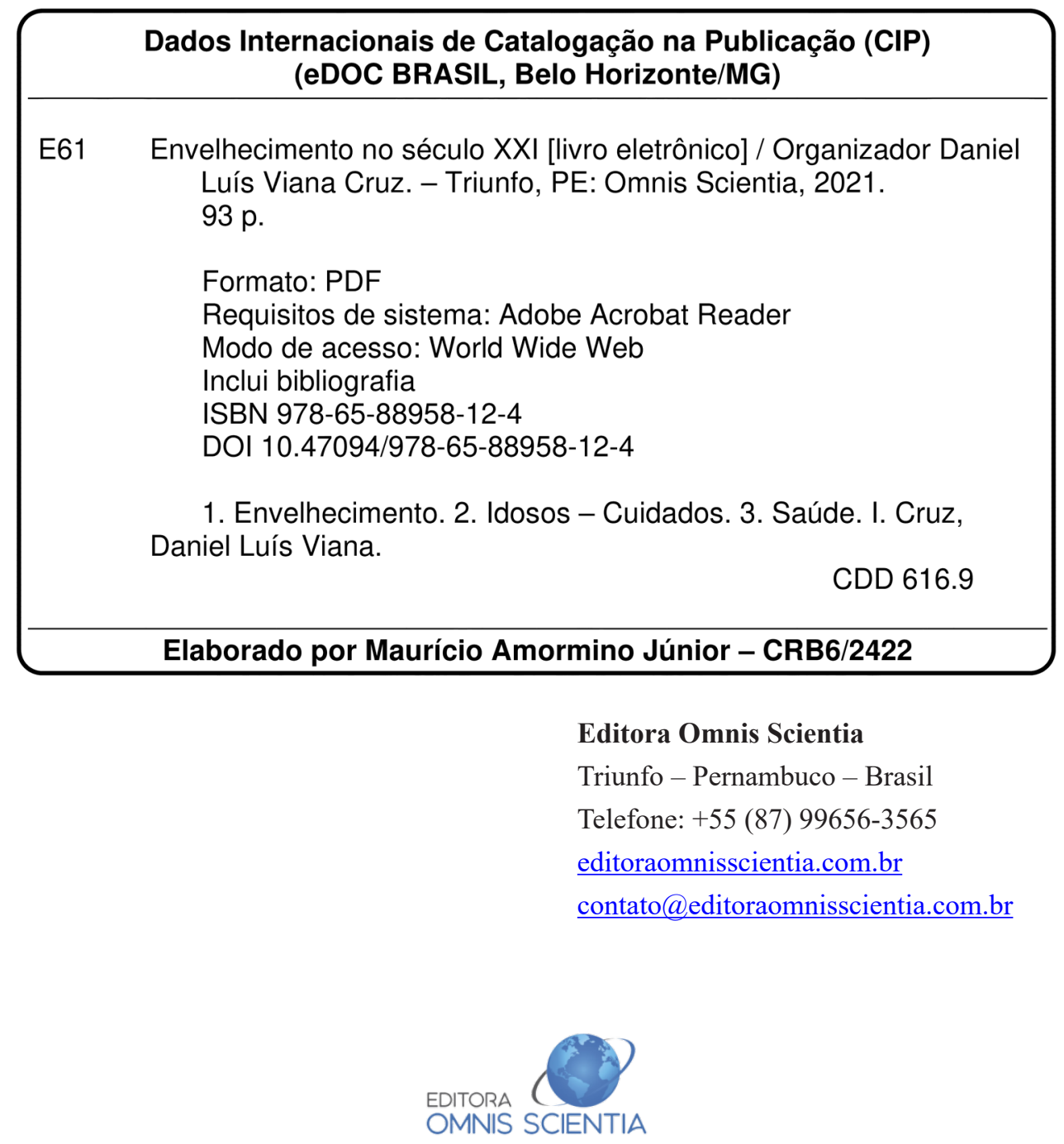




\section{PREFÁCIO}

Sem dúvidas, é provável que a concepção de chegar à terceira idade seja intimidadora para algumas pessoas. Estas sentem diversos medos acerca de problemas de saúde, da solidão, preocupações financeiras e de não ter mais a mesma capacidade de memorização.

Porém, mesmo que haja apreensão em relação ao assunto, deve-se reconhecer que o envelhecimento é um processo inevitável, que ocorre de forma natural na vida de todos os seres vivos. Diante disso, é importante compreender e aceitar essa fase de modo mais positivo. Nessa etapa é importante buscar conhecer os seus direitos, prioridades, limitações e reconhecer que precisa de ajuda de terceiros, de mais atenção em relação à saúde, bem estar e da qualidade de vida.

Os familiares e profissionais que auxiliam os idosos, precisam oferecer uma atenção especial para esses cidadãos, pois nessa fase surgem várias incógnitas para assimilar. Dessa forma, a presente obra aborda temas relacionados à saúde; ao cuidado; às práticas educativas para os idosos e as doenças que os acometem, principalmente para aqueles que vivem em centros de convivência; conhecimento de pessoas da terceira idade sobre o Estatuto do Idoso. Além disso, explana sobre a violência contra o idoso no atendimento hospitalar.

Em nossos livros selecionamos um dos capítulos para premiação como forma de incentivo para os autores, e entre os excelentes trabalhos selecionados para compor este livro, o premiado foi o capítulo 2, intitulado "O CONHECIMENTO DE ADULTOS MAIS VELHOS COM 50 ANOS OU MAIS SOBRE O ESTATUTO DO IDOSO”. 


\section{SUMÁRIO}

CAPÍTULO 1

PROJETOS DE EXTENSÃO: PRÁTICAS EDUCATIVAS PARA IDOSOS

Cássia Rozária da Silva Souza

Antonio Simeone Correia Leitão

Ana Karoline Cordeiro Maia

Yone Almeida da Rocha

Lícia Kellen de Almeida Andrade

Jéssica da Silva Teixeira

Yasmin Maria Pereira Lima

Antonio Hassan da Silva Neto

Maria de Nazaré de Souza Ribeiro

Cleisiane Xavier Diniz

Gabriela Mississipe Correa

DOI: $10.47094 / 978-65-88958-12-4 / 11-18$

CAPÍTULO 2 .19

O CONHECIMENTO DE ADULTOS MAIS VELHOS COM 50 ANOS OU MAIS SOBRE O ESTATUTO DO IDOSO

Wanderson Costa Bomfim

DOI: $10.47094 / 978-65-88958-12-4 / 19-28$

CAPÍTULO 3 .29

CARACTERIZAÇÃO DE IDOSOS VÍTIMAS DE VIOLÊNCIAS ATENDIDOS EM UM HOSPITAL PÚBLICO

Nayara Carolina Mendes 
Mirela Castro Santos Camargos

Cristiano Inácio Martins

Doane Martins da Silva

Karla Rona da Silva

Wanderson Costa Bomfim

DOI: $10.47094 / 978-65-88958-12-4 / 29-38$

CAPÍTULO 4. 39

CONSULTA DE ENFERMAGEM DOMICILIAR A UMA PACIENTE GERIÁTRICA COM HIPERTENSÃO ARTERIAL: UM RELATO DE EXPERIÊNCIA

José Erivelton de Souza Maciel Ferreira

Lilian Brena Costa de Souza

Lídia Rocha de Oliveira

Raiane Martins da Silva

Antônia Hérica Campos Menezes

Lucas Renan Gondim Lopes

Osmar Rodrigues Paixão Neto

Maria Jocelane Nascimento da Silva

Rafaella Martins Mota

Beatriz de Sousa Santos

Marcela de Freitas Matos

Ádria Marcela Vieira Ferreira

DOI: $10.47094 / 978-65-88958-12-4 / 39-50$ 
Rosenda Fernanda Pereira Canavarro

Leslie Bezerra Monteiro

Washington Souza Dos Reis

Raynner Obando De Oliveira

Silvana Nunes Figueiredo

Dayane Jéssyca Cunha de Menezes

DOI: $10.47094 / 978-65-88958-12-4 / 51-62$

CAPÍTULO 6 .63

O USO DE PSICOTRÓPICOS EM IDOSOS

Nicole Kemy Ida Miya1

DOI: $10.47094 / 978-65-88958-12-4 / 63-70$

CAPÍTULO 7.

PRINCIPAIS DOENÇAS QUE ACOMETEM OS PARTICIPANTES DO CENTRO DE CONVIVÊNCIA DE IDOSOS NO MUNICÍPIO DE PEDRO II-PI

Gabriela Barroso Sousa

Ana Paula Pereira da Silva

Andrea Melo Dias

Antônia Layana Araújo

Antônio Victor Pereira do Nascimento

José Ítalo Silva Nascimento

Laiza de Oliveira do Carmo

Lucimary do Nascimento

Yale de Fátima Medeiro Nascimento

DOI: 10.47094/978-65-88958-12-4/71-81 


\section{AVALIAÇÃO DO CARDÁPIO DE INSTITUIÇÃO ASILAR E INFLUÊNCIA NA NUTRIÇÃO}

Déborah Jaqueline Miranda de Moraes Nunes

Ana Letícia Guedes Rocha Barbosa

Ivy Scorzi Cazelli Pires

Lucilene Soares Miranda

Vanessa Alves Ferreira

Bruna Heloísa Miranda de Moraes

DOI: $10.47094 / 978-65-88958-12-4 / 82-90$ 


\title{
O CONHECIMENTO DE ADULTOS MAIS VELHOS COM 50 ANOS OU MAIS SOBRE O ESTATUTO DO IDOSO
}

\author{
Wanderson Costa Bomfim \\ Universidade Federal de Minas Gerais (UFMG) e Instituto René Rachou - Fundação Oswaldo Cruz \\ (FIOCRUZ-MG-IRR), Belo Horizonte, Minas Gerais. \\ http://lattes.cnpq.br/5643648679764089
}

https://orcid.org/0000-0001-7066-2868

RESUMO: Introdução: O Estatuto do Idoso é uma importante legislação para a garantia dos direitos das pessoas com 60 anos ou mais. Entretanto, há uma lacuna na literatura no que tange a análise do conhecimento da população mais envelhecida em relação a essa legislação. Objetivo: Estimar a prevalência de indivíduos que conhecem o Estatuto do Idoso e verificar quais são os seus fatores demográficos e socioeconômicos associados, em indivíduos com 50 anos ou mais, para o Brasil. Metodologia: Trata-se de um estudo transversal, que se utilizou do Estudo Longitudinal de Saúde dos Idosos Brasileiros (ELSI-Brasil). Os resultados foram apresentados por meio de frequências relativas. A associação foi medida por meio do teste qui-quadrado. Resultados: Dentre aqueles que conhecem ou já ouviram falar do Estatuto do Idoso, 18,3\% já leram algo sobre, e 61,5\%, apesar de terem conhecimento, nunca leu o estatuto. Cerca de $20 \%$ não conhecem ou não ouviram falar. Todas as variáveis demográficas e socioeconômicas analisadas foram associadas com o desfecho. Indivíduos mais vulneráveis foram aqueles que tenderam a apresentar menor conhecimento sobre o estatuto, como pessoas mais velhas, não brancas, de menor escolaridade, menor renda e de áreas rurais. Conclusão: Os resultados evidenciam que é necessária uma maior conscientização de todas as pessoas, em especial dos idosos, sobre a existência do estatuto e principalmente sobre seu conteúdo, que garante um leque de direitos que possibilitam melhores condições de vida para as pessoas em idades mais avançadas. Cabe ao poder público garantir que as diretrizes estabelecidas sejam de conhecimento da sociedade e que elas sejam cumpridas na prática.

PALAVRAS-CHAVE: Adultos mais velhos. Estatuto do Idoso. Fatores socioeconômicos.

THE KNOWLEDGE OF OLDER ADULTS 50 YEARS OR OLDER ABOUT THE ELDERLY STATUTE 
ABSTRACT: Introduction: The Elderly Statute is an important legislation to guarantee the rights of people aged 60 or over. However, there is a gap in the literature regarding the analysis of the knowledge of the older population in relation to this legislation. Objective: To estimate the prevalence of individuals who know the Elderly Statute and to verify what are its associated demographic and socioeconomic factors, in individuals aged 50 years or older, for Brazil. Methodology: This is a crosssectional study, which used the Longitudinal Study of Health of the Elderly Brazilians (ELSI-Brazil). The results were presented using relative frequencies. The association was measured using the chisquare test. Results: Among those who know or have heard of the Elderly Statute, 18.3\% have already read about, and $61.5 \%$, despite having knowledge, never read the statute. About 20\% do not know or have not heard of it. All demographic and socioeconomic variables analyzed were associated with the outcome. Most vulnerable individuals were those who tended to have less knowledge about the status, such as older people, non-white, less educated, less income and from rural areas. Conclusion: The results show that it is necessary to raise the awareness of all people, especially the elderly, about the existence of the statute and especially about its content, which guarantees a range of rights that enable better living conditions for people at older ages advanced. It is up to the public authorities to ensure that the guidelines established are known to society and that they are complied with in practice.

KEY WORDS: Older adults. Elderly Statute. Socioeconomic factors.

\section{INTRODUÇÃO}

Os processos de transição epidemiológica e demográfica são marcados por mudanças no perfil de morbimortalidade. As doenças crônicas não transmissíveis e as causadas pelo homem passaram a serem as principais causas de adoecimento e mortalidade, ocorrendo, por sua vez, em idades mais avançadas que em períodos anteriores a essas mudanças (LEE, 2003; ORAN, 2005).

Outra característica desses processos se refere ao envelhecimento populacional e individual. A proporção de idosos vem aumentando, num ritmo acelerado em sociedades de baixa e média renda, incluindo o Brasil (WONG; CARVALHO, 2006; CAMARANO; PASINATO, 2007). Dados do IBGE mostram que em 2043 a população idosa representará $25 \%$ de toda a população (IBGE, 2019). Além disso, as pessoas estão vivendo cada vez mais, como mostrado por estudos que evidenciam o avanço da expectativa ao nascer (CORREA; MIRANDA-RIBEIRO, 2017). Portanto, as sociedades estão cada vez mais envelhecidas.

Esse envelhecimento gera novos desafios enfrentados pelos governos e famílias (MINAYO, 2012). Essas transformações geraram a necessidade de legislações específicas para a garantia de diretos da população idosa. Em nível nacional destaca-se a Política Nacional do Idoso (BRASIL, 1994) e o Estatuto do Idoso (BRASIL, 2003).

O Estatuto do Idoso foi criado em $1^{\circ}$ de outubro de 2003, por meio da lei Lei $\mathrm{n}^{\mathrm{o}} 10.741$, com objetivo de regular os direitos assegurados às pessoas idosas, englobando nesse grupo aqueles com idade igual ou superior a 60 (sessenta) anos (BRASIL, 2003). 
São vários os itens e objetivos estabelecidos pelo Estatuto do Idoso, relacionado aos seus direitos como, a vida, a dignidade e liberdade, moradia, alimentação, saúde e dentre outros (MINSTÉRIO DA SAÚDE, 2013). Entretanto, o que é visto na prática é uma série de desrespeitos às determinações dessa lei, por exemplo, através de situações de violência contra a população mais envelhecida ou problemas associados ao acesso e qualidade dos serviços de saúde demandados (MINAYO, 2005; 2008; PINTO; BARHAM; ALBUQUERQUE, 2013; AMARAL et al., 2012; ALMEIDA et al., 2017).

Apesar da importância dessas e outras leis e normativas, muitas pessoas, incluindo principalmente a população idosa, não conhecem do que se trata o Estatuto do Idoso, o que é estabelecido, e assim, não compreendem os marcos legais existentes capazes de gerar melhorias nas condições de vida desse grupo populacional (MARTINS; MASSAROLLO, 2010).

Diante do exposto, o objetivo do presente estudo estimar a prevalência de indivíduos que conhecem o Estatuto do Idoso e verificar quais são os seus fatores demográficos e socioeconômicos associados, em indivíduos com 50 anos ou mais, para o Brasil.

\section{METODOLOGIA}

O presente estudo tem uma abordagem quantitativa, aplicada e descritiva, que utiliza de dados do Estudo Longitudinal de Saúde dos Idosos Brasileiros (ELSI-Brasil). Esse estudo se refere a uma pesquisa longitudinal, mas que até o presente momento possuiu apenas uma onda de coleta de dados, feita entre os anos de 2015 e 2016, tendo, portanto, um caráter transversal. O ELSI-Brasil é uma pesquisa com representatividade para pessoas com 50 anos ou mais do país e de suas grandes regiões (LIMA-COSTA et al., 2018).

O presente estudo utilizará informações referentes às pessoas com 50 anos ou mais. Apesar do estatuto do idoso se referir as pessoas com 60 anos ou mais, decidiu-se expandir a população alvo, pois ela se refere a indivíduos que irão, num relativo curto espaço de tempo, se tornar legalmente idosos, sendo importante também a análise da percepção desses indivíduos.

A variável de desfecho será o conhecimento da existência do estatuto do idoso. Trata-se de uma variável qualitativa dividida em três categorias: sim e já leu; sim e nunca leu; não conhece ou não ouviu falar. Esse desfecho foi baseado no seguinte quesito da pesquisa: "O (A) $\mathrm{Sr}$ (a) conhece ou já ouviu falar no Estatuto do Idoso?".

Variáveis explicativas demográficas e socioeconômicas foram utilizadas. Dentro das variáveis socioeconômicas foram utilizadas: idade (50 a 59 anos; 60 a 69 anos; 70 a 79 anos e 80 anos e mais); sexo (feminino; masculino); situação conjugal (casado(a)/união consensual/mora junto; solteiro(a); divorciado(a)/separado(a); viúvo(a)); escolaridade (sem instrução; ensino fundamental; ensino médio; ensino superior/pós graduação). Raça (branco; preto; pardo) e renda domiciliar per capta (menos que 1 salário mínimo; 1 salário mínimo; 2 salários mínimos e 3 ou mais salários mínimos). A referência de salário mínimo foi a do ano de 2015 e área (rural e urbano). 


\section{Análise estatística}

Os resultados foram mostrados em termos relativos. A análise univariada dos dados foi baseada em testes qui-quadrado de Pearson para verificar a independência das variáveis e, consequentemente, a associação com o desfecho em questão. As variáveis que foram consideradas independentes, ou seja, que mostraram diferenças nas prevalências do conhecimento sobre o estatuto do idoso entre os grupos em análise, de maneira estatisticamente significativa, foram aquelas que tiveram valor do teste de associação igual ou inferior a 0,05 .

As análises levaram em consideração a amostragem complexa da base de dados, utilizando-se do programa estatístico Stata, versão 13.

\section{Aspectos Éticos}

A pesquisa ELSI-Brasil foi aprovada pelo Comitê de Ética e Pesquisa do Centro de Pesquisas René Rachou da Fundação Oswaldo Cruz (Protocolo no. 886.754).

\section{RESULTADOS E DISCUSSÕES}

Primeiramente é importante a verificação da prevalência de conhecimento do estatuto do idoso. Dentre aqueles que conhecem ou já ouviram falar dessa lei, 18,3\% já leram algo sobre, e $61,5 \%$, apesar de terem conhecimento, nunca leu o estatuto. Cerca de $20 \%$ não conhecem ou não ouviram falar.

As características da população estudada, prevalências e resultados das associações estão descritas na tabela 1. A idade foi associada com o conhecimento da população sobre o estatuto do idoso (p-valor=0,000). Indivíduos mais velhos são aqueles que menos conhecem o estatuto do idoso. Em relação ao status marital, os indivíduos viúvos são aqueles que menos conhecem essa lei (24,9\%). Aqueles solteiros também tiveram um percentual relevante (21,6\%). Essa variável apresentou associação significativa ( $\mathrm{p}$-valor $=0,025)$.

As informações sobre a educação mostram achados importantes e com associação estatisticamente significativa ( $\mathrm{p}$-valor $=0,000$ ). Os indivíduos sem instrução são aqueles com maior percentual de desconhecimento do estatuto do idoso. Cerca de $44 \%$ desses indivíduos não conhecem ou nunca ouviram falar sobre essa legislação e apenas 7,6\% conhecem e já leram. Em contrapartida, para indivíduos com ensino superior ou mais, esses percentuais são 2,3\% e 46,9, respectivamente, evidenciando uma diferença acentuada entre esses grupos extremos.

Outra informação mais socioeconômica importante e com também associação significativa ( $\mathrm{p}$-valor $=0,000)$ é a renda familiar per capta. Assim como para a educação, indivíduos com piores 
condições possuem um menor conhecimento sobre o estatuto do idoso. Para aqueles com renda familiar per capta de 3 salários mínimos ou mais, apenas 9,2\% não conhecem ou nunca ouviram falar sobre o estatuto do idoso. Em contrapartida 38,3\% daqueles que tem a renda de 1 salário mínimo desconhecem a legislação em questão.

A raça também apresentou associação significativa ( $p$-valor=0,002). Os indivíduos autodeclarados brancos apresentaram maior conhecimento sobre o estatuto do idoso, $21,6 \%$ conhecem e já leram essa lei. No que tange a área, há expressiva diferença entre que mora em área urbana e quem mora em área rural. Para o primeiro grupo, 17,8\% desconhecem o estatuto do idoso contra $33,5 \%$ em relação a aqueles que residem em meio rural. Já o conhecimento mais pleno do estatuto também mostra diferenças, pois 19\% dos indivíduos que residem em área urbana conhecem e já leram sobre a legislação em questão, contra apenas 14,6\% daqueles de área rural ( $p$-valor=0,001).

Tabela 1 - Característica da população, análise descritiva e p-valores das análises de associação.

\begin{tabular}{|c|c|c|c|c|c|}
\hline \multirow{2}{*}{ Variáveis } & \multirow{2}{*}{ Total \% } & \multicolumn{3}{|c|}{ Conhecimento sobre Estatuto do idoso } & \multirow{2}{*}{ p-valor } \\
\hline & & sim e já leu & sim e nunca leu & não conhece & \\
\hline Idade & & & & & 0,000 \\
\hline $50-59$ & 47,76 & 17,4 & 64,9 & 17,7 & \\
\hline $60-69$ & 29,64 & 20,3 & 61,4 & 18,3 & \\
\hline $70-79$ & 15,53 & 18,3 & 56,0 & 25,7 & \\
\hline 80 e mais & 7,07 & 16,0 & 50,8 & 33,2 & \\
\hline Sexo & & & & & 0,025 \\
\hline Mulheres & 54,05 & 19,4 & 59,9 & 20,7 & \\
\hline Homens & 45,95 & 17,1 & 63,3 & 19,6 & \\
\hline Estado Civil & & & & & 0,008 \\
\hline Solteiro & 11,5 & 16,2 & 62,2 & 21,6 & \\
\hline Casado/amasiado/união estável & 63,5 & 18,6 & 62,5 & 18,9 & \\
\hline Divorciado(a) ou separado(a) & 10,3 & 21,4 & 58,8 & 19,9 & \\
\hline Viúvo(a) & 14,7 & 16,9 & 58,2 & 24,9 & \\
\hline Escolaridade & & & & & 0,000 \\
\hline Sem Instrução & 13,3 & 7,6 & 48,3 & 44,0 & \\
\hline Fundamental Completo & 59,7 & 13,9 & 64,8 & 21,3 & \\
\hline Médio Completo & 18,7 & 27,4 & 65,4 & 7,2 & \\
\hline Superior ou mais & 8,3 & 46,9 & 50,8 & 2,3 & \\
\hline Renda familiar per capta & & & & & 0,000 \\
\hline Menor que 1 salário mínimo & 46,3 & 14,6 & 61,0 & 24,5 & \\
\hline 1 salário mínimo & 3,8 & 12,3 & 49,5 & 38,3 & \\
\hline 2 salários mínimos & 32,4 & 19,3 & 63,0 & 17,7 & \\
\hline 3 ou mais salários mínimos & 17,5 & 27,7 & 62,7 & 9,5 & \\
\hline Raça/cor & & & & & 0,002 \\
\hline Branco & 42,7 & 21,6 & 62,0 & 16,4 & \\
\hline Preto & 9,7 & 19,1 & 60,8 & 20,1 & \\
\hline Pardo & 44,7 & 16,1 & 61,4 & 22,5 & \\
\hline
\end{tabular}




\begin{tabular}{lccccc} 
Amarela & 1,1 & 9,8 & 68,5 & 21,7 & \\
Indígena & 1,9 & 16,0 & 62,4 & 21,6 & \\
\hline Área & & & & & 0,001 \\
\hline Urbana & 84,7 & 19,0 & 63,2 & 17,8 & \\
Rural & 15,3 & 14,6 & 51,9 & 33,5 & \\
\hline
\end{tabular}

Fonte: ELSI, Brasil, 2015-2016.

O Estatuto do Idoso foi um grande marco no que tange o estabelecimento e proteção dos direitos a pessoa idosa. Trata-se de uma lei que é formada por um conjunto de diretrizes que foram previamente descritas em outros marcos legais como a Constituição Federal, decretos, regulamentos e outros (BOAS, 2015).

Apesar de transcorridos 17 anos do Estatuto do Idoso, sua existência e principalmente seu conteúdo não são triviais para a população idosa e a população em geral, carecendo de amplificação do processo de disseminação para a sociedade, por meio de campanhas educativas que busquem ações intersetoriais (SANTOS et al., 2018).

Alguns estudos buscaram demonstrar a compreensão dos idosos em relação ao Estatuto do Idoso. Em um estudo feito com idosos de uma Unidade Básica de Saúde no município de João Pessoa, evidenciou que metade dos idosos analisados não tinha o conhecimento de alguns direitos básicos, como o atendimento integral prioritário e boa parte desconhece também o direito ao acesso a medicamentos de forma gratuita pela rede pública de saúde (SILVA; COSTA, 2019). Santos e colegas (2018), analisando idosos em municípios do estado do Rio de Janeiro, baseando-se num conjunto de questões, chegaram a conclusão que o Estatuto do Idoso é pouco conhecido por grande parte desses indivíduos.

Há também certo desconhecimento dos direitos como um todo e não apenas do Estatuto do Idoso especificamente. Martins e Massarollo (2010), analisando idosos no município de São Paulo, usando dados dos Núcleos de Convivência de Idosos (NCI), descreveram que 49,2\% dos idosos conheciam seus direitos. Ademais, apenas 25\% disseram que eles são respeitados.

O que se vê na prática é um distanciamento dos direitos estabelecidos por lei e as condições e circunstâncias de vida em que a pessoa idosa é exposta (SILVA, 2008). Um dos direitos assegurados pelo Estatuto do Idoso, em seu artigo n ${ }^{\circ} 15$ é a saúde, que descreve sobre o acesso universal e integral a saúde. O que é visto muitas vezes na prática são dificuldades e barreiras para o acesso e utilização dos serviços, principalmente em determinados grupos de idosos, com piores indicadores socioeconômicos (AMARAL et al., 2012; ALMEIDA et al., 2017).

A alimentação também é outro direito garantido por essa lei. Todavia, estudos mostram elevados percentuais de insegurança alimentar e outros indicadores que representam condições mais precárias de alimentação na população idosa, como ingestão inadequada de nutrientes, em especial para aqueles indivíduos que possuem piores condições socioeconômicas (PREVIDELLI; GOULART; 
A educação possuiu uma relação expressiva com outros fatores socioeconômicos e com a saúde da população idosa. No estatuto do idoso ela é estabelecida no artigo no 20 . Dados do IBGE evidenciam uma melhoria em alguns indicadores de educação no que tange as pessoas com 60 anos ou mais. Em 2000 a taxa de analfabetismo dos idosos era de 39,2\%, reduzindo para 26\% em 2010. Apesar das melhorias, há ainda grandes desigualdades no cenário brasileiro (PERES, 2011). A educação é uma importante ferramenta para o envelhecimento saudável e maior qualidade de vida (MALLMANN et al., 2015).

No estatuto também há o estabelecimento da obrigação da família, em conjunto com a sociedade e do poder público, de assegurar a efetivação dos direitos estabelecidos por lei. Entretanto, o que e visto em muitas circunstâncias, é a família expondo seus membros mais envelhecidos a condições insalubres de vida. Alguns estudos evidenciam experiências de violência no contexto familiar sofridas pelos idosos, cometidas em sua maioria por cônjuges e filhos, mas também pelos seus genros, noras e outros membros. Violências de distintas formas, psicológicas e físicas (MINAYO, 2005; 2008; PINTO; BARHAM; ALBUQUERQUE, 2013; ORGANIZAÇÃO MUNDIAL DA SAÚDE).

Sem dúvidas as últimas décadas foram importantes em termos de legislação que busque a proteção para a população idosa, ocorrendo em conjunto com as mudanças populacionais em curso no país. Entretanto, há muito que ser feito e muitas conquistas ainda são necessárias visando o bem estar dessa população (CAMARANO, 2013).

Este estudo possuiu pontos fortes e fracos. O ponto forte e a realização de uma análise com dados representativos para todo o Brasil no que se refere a pessoas com 50 anos ou mais. Ademais, preenche uma lacuna existente na literatura nacional. Como limitação ressalta-se a não possibilidade de estabelecer uma relação causal entre a variável de desfecho com as demais, pois trata-se de uma abordagem transversal. Como pesquisa futura ressalta-se a importância de uma análise por meio de modelos estatísticos, para a exploração dos efeitos dos fatores demográficos e socioeconômicos, investigados conjuntamente, no desfecho em questão.

\section{CONCLUSÃO}

O presente estudo evidenciou um considerável desconhecimento dos adultos mais velhos em relação ao Estatuto do Idoso. O maior percentual, apesar de ter sido daqueles que conhecem ou já ouviram falar dessa legislação, foi também de pessoas que nunca o leu, evidenciando um conhecimento bastante superficial. Indivíduos mais vulneráveis foram aqueles que tenderam a apresentar menor conhecimento sobre o estatuto, como pessoas mais velhas, não brancas, de menor escolaridade, menor renda e de áreas rurais.

Os resultados evidenciam que é necessária uma maior conscientização de todas as pessoas, em especial dos idosos, sobre a existência do estatuto e principalmente sobre seu conteúdo, que garante 
um leque de direitos que possibilitam melhores condições de vida para as pessoas em idades mais avançadas. Cabe ao poder público garantir que as diretrizes estabelecidas sejam de conhecimento da sociedade e que elas sejam cumpridas na prática.

\section{AGRADECIMENTOS}

Agradeço o Conselho Nacional de Desenvolvimento Científico e Tecnológico (CNPq) pelo apoio no desenvolvimento deste estudo.

\section{REFERÊNCIAS}

ALMEIDA, A.P.S.C et al . Determinantes socioeconômicos do acesso a serviços de saúde em idosos: revisão sistemática. Rev. Saúde Pública, São Paulo, v. 51, 50, 2017.

AMARAL, F.L.J.S et al . Fatores associados com a dificuldade no acesso de idosos com deficiência aos serviços de saúde. Ciênc. saúde coletiva, Rio de Janeiro, v. 17, n. 11, p. 2991-3001, Nov. 2012

BOAS, M.A.V. Estatuto do Idoso Comentado.5 Edição. 2015. Editora Forense.

BRASIL. LEI No 8.842, DE 4 DE JANEIRO DE 1994. Dispõe sobre a política nacional do idoso, cria o Conselho Nacional do Idoso e dá outras providências.

BRASIL. LEI No 10.741, DE $1^{\circ}$ DE OUTUBRO DE 2003. Dispõe sobre o Estatuto do Idoso e dá outras providências.

CAMARANO, A.A. Estatuto do idoso: Avanços com contradições. IPEA. 2013.

CAMARANO, A.A; PASINATO, M.T. Envelhecimento, pobreza e proteção social na América Latina. Pap. poblac, Toluca, v. 13, n. 52, p. 9-45, jun. 2007.

CORREA, É.R.P.; MIRANDA-RIBEIRO, A. Ganhos em expectativa de vida ao nascer no Brasil nos anos 2000: impacto das variações da mortalidade por idade e causas de morte. Ciênc. saúde coletiva. v. 22, n. 3, p. 1005-1015. 2017.

DUARTE VENTURINI, C., et al. Inadequate food intake in elderly: drug-nutrient interaction. PAJAR - Pan American Journal of Aging Research, v.8, n.1 e34072.2020.

INSTITUTO BRASILEIRO DE GEOGRAFIA E ESTATÍSTICA. IBGE. Idosos indicam caminhos para uma melhor idade. Disponível em: < https://censo2021.ibge.gov.br/2012-agencia-denoticias/noticias/24036-idosos-indicam-caminhos-para-uma-melhor-idade.html\#: :text=A\%20 popula $\%$ C3\%A7\%C3\%A3o\%20idosa $\% 20$ tende $\% 20$ a,do $\% 20$ IBGE $\% 2 C \% 20$ atualizada $\% 20 \mathrm{em} \% 20$ 2018.\&text $=\mathrm{A} \% 20$ rela $\% \mathrm{C} 3 \% \mathrm{~A} 7 \% \mathrm{C} 3 \% \mathrm{~A} 3 \mathrm{o} \% 20$ entre $\% 20 \mathrm{a} \% 20$ porcentagem, $\% 2 \mathrm{C} 47 \% 25 \% 2 \mathrm{C} \% 20$ 
em\%202060.>. Acesso em: Novembro. 2020.

LEE, R 2003. The Demographic Transition: Three Centuries of Fundamental Change. Journal of Economic Perspectives, v.17,n.4,p. 167-190.2003.

LIMA-COSTA, M.F, et al. The Brazilian Longitudinal Study of Aging (ELSI-Brazil): Objectives and Design. Am J Epidemiol. v.187, n.7, p.1345-1353. 2018.

MALLMANN, D.G.et al . Educação em saúde como principal alternativa para promover a saúde do idoso. Ciênc. saúde coletiva, Rio de Janeiro, v. 20, n. 6, p. 1763-1772, June 2015.

MARTINS, M. S.; MASSAROLLO, M.C. K. B. Conhecimento de idosos sobre seus direitos. Acta paulista de Enfermagem, v. 23, n. 4, p. 479-485, 2010.

MARTINS, M.S; MASSAROLLO; M.C.K.B. Conhecimento de idosos sobre seus direitos. Acta paul. enferm., São Paulo, v. 23, n. 4, p. 479-485, 2010 .

MINAYO, M.C.S. O envelhecimento da população brasileira e os desafios para o setor saúde. Cad. Saúde Pública. v. 28, n. 2, p. 208-210. 2012.

MINAYO, M. C. S. Violência e maus-tratos contra a pessoa idosa: é possível prevenir e superar. In T. Born (Ed.), Cuidar melhor e evitar a violência: manual do cuidador da pessoa idosa (pp.38-45). Brasília, DF: Secretaria Especial dos Direitos Humanos. 2008.

MINAYO, M. C. S. Violência: um velho-novo desafio para a atenção à saúde. Revista Brasileira de Educação Médica. v.29, n.1, p. 55-63. 2005.

OMRAN, A.R. The Epidemiologic Transition: A Theory of the Epidemiology of Population Change. Milbank Q. v.83,n.4, p.731-757.

ORGANIZAÇÃO MUNDIAL DA SAÚDE -OMS. Violence against women: Globalpicture health response. Geneva, Switzerland: Department ofReproductive Health and Research, World Health. 2013a.

ORGANIZAÇÃO MUNDIAL DA SAÚDE -OMS. Department of gender, women and health, family and community health. Adressing violence against women and achieving the milennium goals. Geneva: World Health Organization; 2005.

PERES, M.A.C. Velhice e analfabetismo, uma relação paradoxal: a exclusão educacional em contextos rurais da região Nordeste. Soc. estado., Brasília, v. 26, n. 3, p. 631-662, Dec. 2011.

PINTO, F.N.F.R.; BARHAM, E.J; ALBUQUERQUE, P.P. Idosos vítimas de violência: fatores sociodemográficos e subsídios para futuras intervenções. Estud. pesqui. psicol. v. 13, n. 3, p. 11591181.2013 . 
PREVIDELLI, A.N; GOULART, R.M.M; AQUINO, R.C. Balanço de macronutrientes na dieta de idosos brasileiros: análises da Pesquisa Nacional de Alimentação 2008-2009. Rev. bras. epidemiol., São Paulo, v. 20, n. 1, p. 70-80, Mar. 2017.

SANTOS, E.N. et al. Crenças De Idosos Em Relação Ao Estatuto Do Idoso. Lex Humana, Petrópolis, v. 10, n. 2, p. 14-40, 2018.

SILVI, A.C.A.P. Conhecimento, Cidadania e Direito do Idoso: relatos pós-Lei no 10.741/2003. Rev.

Bras. Geriatr. Gerontol. v.11, n.1, p.45-5. 2008.

SILVA, M.S.L.; COSTA, S.M.G. Percepção Da Pessoa Idosa Acerca Do Estatuto Do Idoso. VI Congresso Internacional de Envelhecimento Humano. 2019.

WONG, Laura L. Rodríguez; CARVALHO, J. A.. O rápido processo de envelhecimento populacional do Brasil: sérios desafios para as políticas públicas. Rev. bras. estud. popul., São Paulo, v. 23, n. 1, p. 5-26. 2006 . 


\section{ÍNDICE REMISSIVO}

A

acadêmicos de enfermagem 12, 14, 43

adequação de nutrientes 81

agressões $17,30,31,34,35,36,37,59$

Alimentação 27, 82, 84

atividade física $12,46,47,62,64,65,66,67,79,87,88$

atividades de lazer 51, 59

Avaliação Geriátrica 41, 43

Avaliação Nutricional de idosos 82

AVC 71,72

B

baixa autoestima 51,58

C

Centro de Convivência de Idosos 71, 72

comorbidade psiquiátrica 62,63

condicionamento físico 71,73

condições somáticas 62,63

conhecimento $12,13,15,19,21,22,23,24,25,41,46,47,48,49,52,53$

conhecimento da sociedade 19,26

conscientização 19,25

cuidado ao idoso $51,57,59$

cuidado nutricional 82,87

cuidadores de idosos 51

\section{D}

desnutrição $67,81,83,86,87,88$

diabete 71,74

Doença de Alzheimer 50, 53, 54, 55, 56, 57, 58, 59, 60, 61

doenças crônicas 20, 41, 42, 49, 66

$\mathbf{E}$

efeitos colaterais 62,64

envelhecimento $12,16,20,25,27,28,31,37,41,42,62,65,67,69,72,74,81,83,86,88$

envelhecimento ativo 12,16 
esgotamento físico 51,58

estado nutricional $45,82,83,84,88$

Estatuto do Idoso 19, 20, 21, 24, 25, 26, 32, 35, 36

expectativa de vida 26,71

experiência vivenciada 12

$\mathbf{F}$

fases da vida 62

G

grau de vulnerabilidade 81,83

grupo etário 48, 62, 63

$\mathbf{H}$

hipertensão 41, 43, 45, 47, 49, 66, 71, 72, 73, 74, 78

hipertensão arterial $41,43,47,49,72$

hospital público 29, 31, 32, 33, 34, 35, 36, 37

I

idoso institucionalizado 82,87

idosos $14,15,16,17,19,20,21,24,25,26,27,29,31,32,33,34,35,36,37,38,41,42,47,48,49,50,51,52,53,55$, $56,57,58,59,60,62,63,64,65,66,67,68,71,72,73,74,78,79,81,83,84,86,87,88,89$

imunidade 71

instituição asilar 82,83

Instituições de Longa Permanência para Idosos (ILPI) 81, 83

instruções 12

internação hospitalar 30, 34, 35

isolamento social $17,51,58$

$\mathbf{L}$

Lar para Idosos 82,83

legislação 19, 22, 23, 25, 49

lesões $30,35,38,45$

M

macro nutriente $82,84,85,86$

$\mathbf{N}$

necessidades do idoso 41, 43

Nutrição $82,84,87$ 
nutrição adequada $62,64,66,86$

$\mathbf{0}$

obesidade $66,74,81$

$\mathbf{P}$

paciente geriátrica $41,43,44,45,46$

pandemia $12,15,16,17$

patologias $41,42,48$

patologias crônicas degenerativas 41, 42

pessoas mais velhas 19,25

políticas públicas do idoso 62,65

população idosa $12,16,20,21,24,25,31,32,35,36,62,64,65,73,75,79$

práticas educativas $12,16,18$

Práticas Educativas com Idosos 12, 14

problema de saúde $30,31,34$

Projeto de Extensão 12, 14

projeto de integração 71

promoção à saúde 12,14

promoção da saúde 62,75

pronto-socorro 29

psicofármacos $62,63,64,66,68$

psicotrópicos $62,63,64,65,66,67,68$

Q

qualidade de vida $13,14,25,30,34,35,41,48,52,53,55,59,60,62,65,66,72,79,83,88$

S

saúde $12,13,14,15,16,17,18,21,24,25,26,27,30,31,34,35,36,37,41,42,43,46,47,48,49,51,52,55,56,57$, $58,59,62,63,64,65,66,67,68,71,73,74,75,78,79,82,83,84,87,88,89$

saúde do idoso $17,27,62,65,68$

Saúde dos Idosos 19, 21

$\mathbf{T}$

Tecnologia educacional 12

terapia auricular $62,65,66,67$

terceira idade $62,71,72$

traumas 30,35 
vídeos educativos 12,16

violência $21,25,27,29,31,32,33,34,35,36,37,38$

vítimas de agressão $30,32,36$

vítimas de violência $29,31,34$ 
